\title{
(2) \\ An Ethnobotanical Study of Medicinal Plants in Asgede Tsimbila District, Northwestern Tigray, Northern Ethiopia
}

\author{
G. Zenebe, M. Zerihun, and Z. Solomon
}

\section{Research}

\begin{abstract}
Investigation and documentation of the status of medicinal plants and associated knowledge was conducted in Asgede Tsimbila district, northwestern Tigray, northern Ethiopia. Data was collected and evaluated with a questionnaire survey, semi-structured interviews, field observations, direct matrix ranking, preference ranking, abundance scores, and vegetation surveys. Sixty-eight medicinal plant species used to treat 50 different ailments (in humans and livestock) were recorded. Leaves are the most commonly collected plant parts for medicinal purposes. Much of the ethno-medicinal knowledge is concentrated in elderly members of the community. The medicinal plants are facing threats from agricultural expansion, wood extraction and overgrazing. Consequently, abundance of medicinal plant resources is declining with time. Furthermore, effort to conserve and cultivate medicinal plants is virtually non-existent. Thus, participation of the local people and awareness creation on sustainable utilization and management of these resources is vital.
\end{abstract}

\section{Introduction}

About $80 \%$ of Ethiopian people rely on traditional medicine to meet their health care needs (Bekele 2007). The wide spread use of traditional medicine could be attributed to cultural acceptability, perceived efficacy against certain types of diseases, physical accessibility and affordability as compared to modern medicine (Bekele 2007, Defar 1998, Hunde et al. 2006). Nevertheless, little effort has so far been made to properly document the associated knowledge base and conserve medicinal plants in the country (Gidey et al. 2009). Even though encouraging initiatives have emerged in recent years, studies conducted hitherto are far from complete owing to the multi-ethnic cultural diversity and the diverse flora of Ethiopia (Bekele 2007, Yineger et al. 2008). Medicinal plants and the asso- ciated knowledge are being threatened by ongoing deforestation, environmental degradation and 'modernization' (Balemie et al. 2004, Bekele 2007). All this necessitates the need to investigate the status of medicinal plant resources and knowledge base associated with it for successful resource conservation and development.

The present study was, therefore, initiated in Asgede Tsimbila district, northwestern Tigray, northern Ethiopia in a context where medicinal plants suffer notable disregard from research and development strategies. The objective of the study was to investigate and document the status, trends in abundance and threats to medicinal plants, and the associated indigenous knowledge in the study area.

\section{Materials and Methods}

\section{Description of the study area}

The study was carried out in northwestern part of Tigray Regional State, northern Ethiopia, in Asgede Tsimbila dis-

\section{Correspondence}

Girmay Zenebe, Hawassa University, Wondo Genet College of Forestry and Natural Resources, School of Forestry, P. O. Box 128, Shahemene, ETHIOPIA. zenebegirmay@gmail.com Mohammed Zerihun, Forum for Social Studies, Addis Ababa, ETHIOPIA. zerihunmohammed@yahoo.com

Zewdie Solomon, Hawassa University, Wondo Genet College of Forestry and Natural Resources, School of Forestry, P. O. Box 128, Shahemene, ETHIOPIA. zew172@yahoo.com.

Ethnobotany Research \& Applications 10:305-320 (2012)

Published: July 25, 2012 
trict $\left(14^{\circ} 42^{\prime}\right.$ to $14^{\circ} 11^{\prime} \mathrm{N}$ and $37^{\circ} 34^{\prime}$ to $\left.38^{\circ} 19^{\prime} \mathrm{E}\right)$ (Figure 1). The district covers a total surface area of about 239,955 ha, which is $4.4 \%$ of the total Tigray regional area (Genzebu 2009). The agro-climatic zone of the district is characterized by hot to warm semi-arid lowlands, hot to warm sub-moist lowlands and tepid to cool sub-moist mid highlands. The area has a unimodal rainfall pattern (June to September) with a total annual rainfall ranging between 500 and $750 \mathrm{~mm}$. The mean annual temperature ranges between 20 and $35^{\circ} \mathrm{C}$ with an altitudinal range of 800 to 2300 m.a.s.l.

The vegetation cover of the area can be grouped into four major plant communities: Acacia-Commiphora woodland, Combretum-Terminalia woodland, Riparian woodland and Dry-evergreen mountain woodlands. The district has 23 rural kebeles (rural sub-districts- the smallest administrative unit next to district in Ethiopia) with a total population of about 128,363 . The livelihood of the local people is mainly based on subsistence mixed agriculture (croplivestock production) (Kahsay 2007).

\section{Selection of study sites and informants}

A reconnaissance survey was first conducted to have an overview of the demographic, socio-economic and bio-physical conditions of the study area. Development agents of the district and elders were contacted during the survey. The preliminary survey results indicated that the kebeles are essentially similar to one another. Consequently, three study site kebeles were randomly selected: Alogien, Lemlem and Hintset. Simple stratified random sampling (based on age and sex) was employed to select informants. Accordingly, a total of 72 respondents (36 males and 36 females) stratified into two age classes (below 40 and above 40 years) were selected from the study sites constituting about $5 \%$ of the total population. Out of these, 20 key informants (12 males and 8 females) were selected as recommended by local knowledgeable elders.

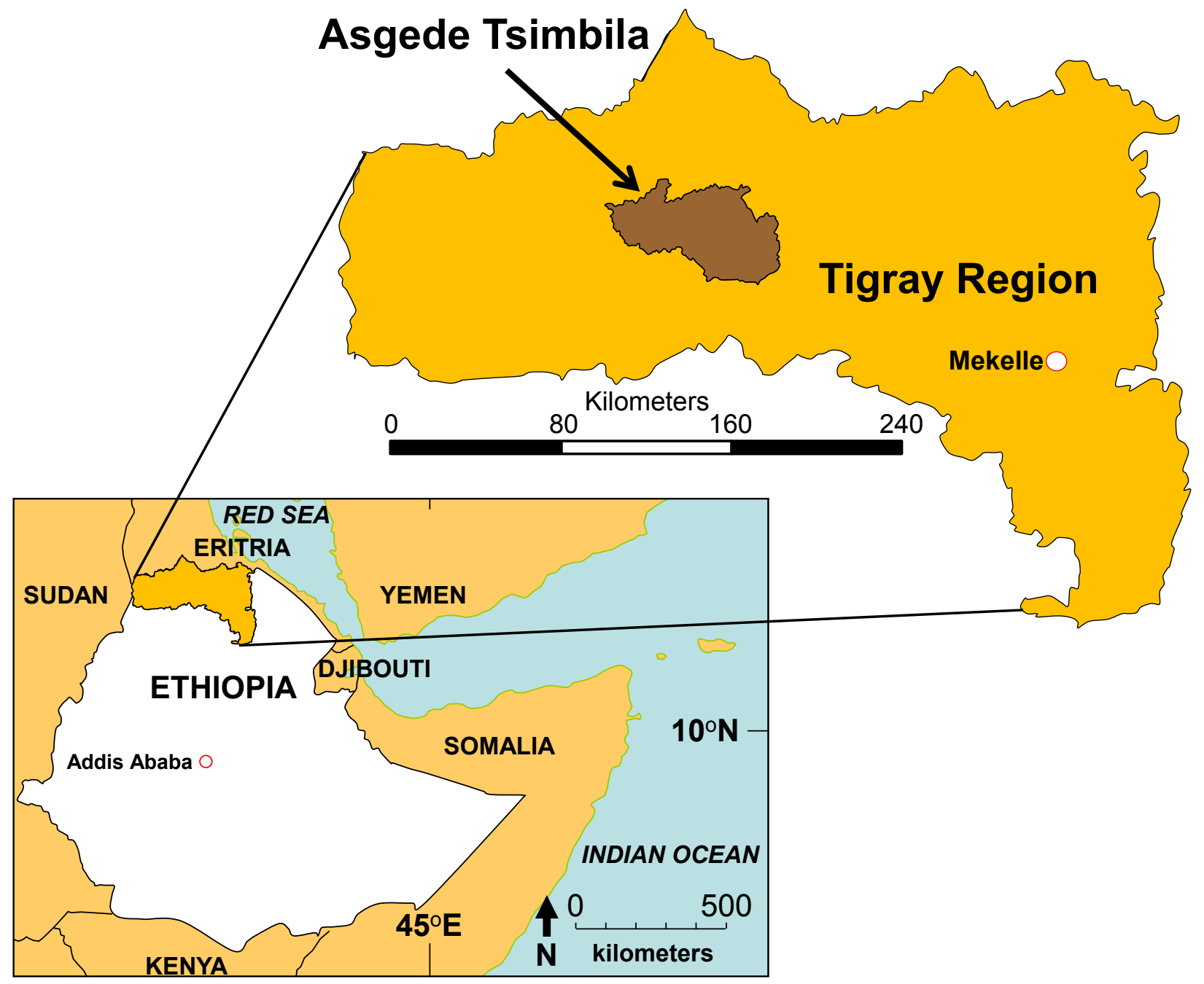

Figure 1. Study site in Asgede Tsimbila District, Tigray Region, Ethiopia. 


\section{Authors - An Ethnobotanical Study of Medicinal Plants in Asgede Tsimbila District, Northwestern Tigray, Northern Ethiopia}

\section{Data collection}

\section{Ethnobotanical data}

Ethnobotanical data were collected between June and September 2010 using a questionnaire survey, semistructured interviews with key informants (knowledgeable elders, religious leaders and other individuals), field observation, and ranking and scoring methods (Cotton 1996, Martin 1995, Nanyunja 2003). Interviews and discussions were conducted in Tigrigna (the local language) using a checklist of topics. At the start of each interview the aim of the interview was explained to invoke clear and objective responses and then informants were asked for their consent. A range of ethnobotanical information including plant local names, uses, parts used, mode of utilization, plant habit and habitat, conservation status, trends in abundance of the species and existing threats to the plants were covered. Ethnobotanical surveys of local knowledge can be used as a tool for investigating human perceptions of biodiversity loss (change in abundance and use of plant species through time) (Nanyunja 2003). This technique enables us to tap people's memories, to recall important historical events which have taken place in a given area (Nanyunja 2003). Hailemariam et al. (2009) have also confirmed that ethnobotanical data collection tools can be used to glean information related with the conservation status and cultivation practices of medicinal plants. In a similar vein, this study has emphasized on the indigenous knowledge of the local people to investigate the conservation status and/or abundance of medicinal plants, and the possible causes of these changes. Vegetation surveys and field observations (through transect walks) were also conducted to corroborate these results.

\section{Direct matrix and preference ranking}

Direct matrix ranking was exercised for seven commonly reported multipurpose medicinal plants in order to assess their relative importance to local people (Martin 1995). Based on their relative uses, selected informants were asked to assign use values for each plant $(5=$ best, $4=$ very good, 3 = good, 2 = less used, 1 = least used and 0 $=$ not used) to each use category. Use categories in the comparison include construction, medicine, fruit, fodder, fuel wood, shade and fence.

Preference ranking was also conducted to determine the relative importance of six commonly reported medicinal plants used in the treatment of stomach pain/'abdominal disorder', a common ailment in the district (Martin 1995). The traditional healers in the locality do treat livestock although the frequency of livestock disease is not comparable to human illness. Six traditional healers were given specimens of the medicinal plants and asked to arrange them based on their personal preference of efficacy. The medicinal plant supposedly most effective was given the highest score (6), while the one with the least effectiveness given the lowest score (1).

\section{Threats to and trends in abundance of medicinal plants}

In the study area preliminary investigation identified various human induced and natural factors possibly threatening the survival of medicinal plants. Five threatening factors were considered in this ranking: agricultural expansion, drought incidence, fire wood extraction, overgrazing, and construction. The relative importance of the threatening factors was ranked using priority ranking (Martin 1995). Priority ranking was based on the level of destructive effects of each threatening factor (1 to 5 scores were assigned where one is for the least and five the most destructive threat). Furthermore, abundance scores were established for some of the most commonly reported and valuable medicinal plants in the study area for the period 1970 to 2010 . The procedure of abundance scores is adopted from Nanyunja (2003). The scores were used to reveal the trends in abundance of the selected species in the given time span. The scores used ranged from 0 to 2, where 0 is = none or almost none; 1 means a few or some; and 2 is for many.

\section{Vegetation data and plant identification}

Vegetation surveys were carried out both in homesteads and in the wild to assess the distribution of the most frequently reported plant species. A total of 18 quadrat plots (20 m x $20 \mathrm{~m}$ ) were systematically established in the wild (12 quadrats) and in homesteads (6 quadrats) following the method of Tolassa (2007). The plots covered all habitat types occurring in the area: rocky/hilly areas, flat top mountain/plateaus, valleys and riverine areas, and plain areas. The homogeneity of each tree stand was checked through observation before laying down a sample plot. Three quadrats were established in each of the aforementioned habitat types. The remaining six quadrats were established by random identification of two homesteads from each study site. Then, counts of each species (presence or absence) were conducted in each quadrat.

Specimens of medicinal and other plants found to be major components of the vegetation of the district were collected and crosschecked for their local names with the help of key informants and development agents. Botanical names were established by comparing specimens with those at the National Herbarium, Science Faculty, Addis Ababa University using available floras (Bekele-Tesemma et al. 1993, Edwards et al. 1995, 1997, 2000, Fichtl \& Admasu 1994, Hedberg \& Edwards 1989, 1995, Hedberg et al. 2004, 2006, November et al. 2002).

\section{Data analysis and presentation}

Triangulation was employed to evaluate the validity of information collected using different surveying methods 
(Mintsa-Mi Nzue 2009). Then, the data collected using different ethnobotanical methods such as questionnaire survey and interviews, along with the data in the form of scores were organized, entered and analyzed in Microsoft Excel. Data were subjected to descriptive analysis and percentages were generated. The data from ranking methods (direct matrix ranking, preference/priority ranking) were presented in the form of ranks where ranks were determined based on the total scores under each attribute. Pearson's correlation test was also run in SPSS 16.0 software (SPSS 2008) to find out the relationship between knowledge distribution by age and sex of respondents (Yineger \& Yewhalaw 2007). Frequency and relative frequency of plant species (Martin 1995) were calculated for the vegetation data.

\section{Results}

\section{Traditional medicinal plants reported and associated knowledge}

The informants reported 68 plant species as medicinally important (Table 1). The plants belong to 44 families and 64 genera, with Euphorbiaceae and Fabaceae (six species each), and Cucurbitaceae and Solanaceae (three species each) being the most represented plant families.

\section{Habitat, growth forms and plant parts used}

\section{Habitat}

In the study area, majority of the medicinal plants (69\%) were reported to be collected from the wild, $24 \%$ are do-

Table 1. Medicinal plants used to treat human/livestock ailments in Asgede Tsimbila District, Tigray Region, Ethiopia. Habits: $\mathrm{T}=$ tree, $\mathrm{Sh}=$ shrub, $\mathrm{H}=$ herb, $\mathrm{C}=$ climber. Parts used: $\mathrm{R}=$ root, $\mathrm{L}=$ leaf, $\mathrm{F}=$ fruit, $\mathrm{FI}=$ flower, $\mathrm{B}=\mathrm{bark}, \mathrm{ME}=\mathrm{milky}$ exudate, $\mathrm{Yt}=$ young twig. $\mathrm{FMD}=$ foot and mouth disease (livestock ailment)

\begin{tabular}{|c|c|c|c|c|c|}
\hline Medicinal plants [Family] & Local name & Habit & Diseases treated & $\begin{array}{l}\text { Parts } \\
\text { used }\end{array}$ & $\begin{array}{l}\text { Voucher } \\
\text { number }\end{array}$ \\
\hline $\begin{array}{l}\text { Acokanthera schimperi (A. DC.) Benth. \& } \\
\text { Hook. f. [Apocynaceae] }\end{array}$ & Mebtie & Sh & $\begin{array}{l}\text { Insecticide, anti-snake/ } \\
\text { mouse }\end{array}$ & $L$ & ZG-027 \\
\hline $\begin{array}{l}\text { Agave sisalana Perrine ex Engelm. } \\
\text { [Agavaceae] }\end{array}$ & Eka & $\mathrm{H}$ & Ear disease & $\mathrm{S}$ & ZG-011 \\
\hline Albuca abyssinica Jacq. [Asparagaceae] & $\begin{array}{l}\text { Shingurti- } \\
\text { zibei }\end{array}$ & $\mathrm{H}$ & Elephantiasis & $\mathrm{R}$ & ZG-054 \\
\hline Allium sativum L. [Amaryllidaceae] & $\begin{array}{l}\text { Tsaeda- } \\
\text { shingurti }\end{array}$ & $\mathrm{H}$ & Herpes virus, cough & $\mathrm{R}$ & ZG-066 \\
\hline Aloe vera (L.) Burm.f. [Xanthorrhoeaceae] & Ere & $\mathrm{H}$ & Sprain & $\mathrm{R}$ & ZG-028 \\
\hline $\begin{array}{l}\text { Anogeissus leiocarpa (DC.) Guill. \& Perr. } \\
\text { [Combretaceae] }\end{array}$ & Hanse & $\mathrm{T}$ & $\begin{array}{l}\text { Stomach pain, } \\
\text { diarrhoea, malaria, } \\
\text { wounds }\end{array}$ & $B, L$ & ZG-032 \\
\hline Azadirachta indica A. Juss. [Meliaceae] & Nim & $\mathrm{T}$ & $\begin{array}{l}\text { Anti-termite, petriasis } \\
\text { versicolor, leech } \\
\text { infestation, hen disease }\end{array}$ & $\mathrm{L}$ & ZG-001 \\
\hline $\begin{array}{l}\text { Balanites aegyptiacus (L.) Delile } \\
\text { [Xygophyllaceae] }\end{array}$ & Mekie & $\mathrm{T}$ & $\begin{array}{l}\text { Abdominal problems, } \\
\text { tuberculosis }\end{array}$ & $\mathrm{F}$ & ZG-008 \\
\hline Boscia angustifolia A. Rich. [Capparaceae] & Kermed & $\mathrm{T}$ & Evil sprit & Yt & ZG-044 \\
\hline $\begin{array}{l}\text { Boswellia papyrifera (Del.) Hochst. } \\
\text { [Burseraceae] }\end{array}$ & Meker & $\mathrm{T}$ & Diarrhea & B & ZG-014 \\
\hline Brassica rapa L. [Brassicaceae] & Hamli-adri & $\mathrm{H}$ & Wounds & L & ZG-050 \\
\hline $\begin{array}{l}\text { Bridelia micrantha (Hochst.) Baill. } \\
\text { [Phyllanthaceae] }\end{array}$ & Abetere & $\mathrm{T}$ & Scorpion bite & B & ZG-003 \\
\hline $\begin{array}{l}\text { Buddleja polystachya Fresen. } \\
\text { [Scrophulariaceae] }\end{array}$ & Metere & $\mathrm{T}$ & Headache, migrain & Yt & ZG-040 \\
\hline $\begin{array}{l}\text { Calotropis procera (Aiton) W.T. Aiton } \\
\text { [Apocynaceae] }\end{array}$ & Gindiae & Sh & $\begin{array}{l}\text { Warts, swelling, } \\
\text { inflammatory wounds }\end{array}$ & ME & ZG-012 \\
\hline Calpurnia aurea (Aiton) Benth. [Fabaceae] & Hitsawutse & Sh & $\begin{array}{l}\text { Swelling, mange, } \\
\text { tuberculosis, insecticide }\end{array}$ & $\begin{array}{l}\mathrm{R} / \mathrm{L} \\
\mathrm{F}\end{array}$ & ZG-030 \\
\hline
\end{tabular}


Authors - An Ethnobotanical Study of Medicinal Plants in Asgede Tsimbila

District, Northwestern Tigray, Northern Ethiopia

\begin{tabular}{|c|c|c|c|c|c|}
\hline Medicinal plants [Family] & Local name & Habit & Diseases treated & $\begin{array}{l}\text { Parts } \\
\text { used }\end{array}$ & $\begin{array}{l}\text { Voucher } \\
\text { number }\end{array}$ \\
\hline Capparis tomentosa Lam. [Capparaceae] & Andel & Sh & $\begin{array}{l}\text { Epilepsy, evil eye, } \\
\text { evil sprit }\end{array}$ & $\begin{array}{l}\text { R, B, } \\
S\end{array}$ & ZG-018 \\
\hline Carica papaya L. [Caricaceae] & Papayo & $T$ & Herpes virus, amoebas & $\begin{array}{l}\text { L/FI, } \\
\mathrm{F}\end{array}$ & ZG-048 \\
\hline Carissa edulis (Forssk). Vahl [Apocynaceae] & Agam & Sh & Evil eye, epilepsy & $R$ & ZG-029 \\
\hline Cissus petiolata Hook.f. [Vitaceae] & Alkie & $\mathrm{C}$ & $\begin{array}{l}\text { Mange, (FMD), snake } \\
\text { bite, swelling of cattle, } \\
\text { back pain in cattle }\end{array}$ & $S, L$ & ZG-036 \\
\hline Citrus limon (L.) Burm.f. [Rutaceae] & Lemin & Sh & \begin{tabular}{|l} 
Cough, herpes \\
virus, tuberculosis \\
(abay seal)
\end{tabular} & $F, L$ & ZG-060 \\
\hline $\begin{array}{l}\text { Clerodendrum myricoides (Hochst.) R. Br. ex } \\
\text { Vatke [Lamiaceae] }\end{array}$ & Surbetri & Sh & \begin{tabular}{|l} 
Sprain, headache, \\
Epilepsy, Bovine \\
pasteurellosis, \\
Babesiasis
\end{tabular} & $\begin{array}{l}\text { S, R, } \\
\text { L, S }\end{array}$ & ZG-053 \\
\hline Combretum sp. [Combretaceae] & Akuma & $\mathrm{T}$ & $\begin{array}{l}\text { Abdominal problems/ } \\
\text { diarrhea, stomach pain }\end{array}$ & $\mathrm{B} / \mathrm{R}$ & ZG-022 \\
\hline Cordia africana Lam. [Boraginaceae] & Awhi & $T$ & $\begin{array}{l}\text { Migraine, broken } \\
\text { body/hand, wounds, } \\
\text { gastritis/constipation }\end{array}$ & $\mathrm{B} / \mathrm{L}, \mathrm{F}$ & ZG-039 \\
\hline $\begin{array}{l}\text { Croton macrostachyus Hochst. ex Delile } \\
\text { [Euphorbiaceae] }\end{array}$ & Tambok & $\mathrm{T}$ & \begin{tabular}{|l|} 
Rabies, \\
spleenomegally, \\
Ovine pasteurellosis, \\
epilepsy, anti-termite, \\
Hyper-blurbia \\
\end{tabular} & $\begin{array}{l}\mathrm{B}, \mathrm{S} \\
\mathrm{R}, \mathrm{L}\end{array}$ & ZG-027 \\
\hline Cucumis dipsaceus Ehrenb. [Cucurbitaceae] & Hafaflo & C & $\begin{array}{l}\text { Snake bite, insecticide, } \\
\text { stomach pain/ } \\
\text { diarrhea, tuberculosis }\end{array}$ & $R$ & ZG-037 \\
\hline Cucurbita maxima Duchesne [Cucurbitaceae] & Duba & $\mathrm{C}$ & Tape worm & $\mathrm{F}$ & ZG-041 \\
\hline Cucurbita pepo L. [Cucurbitaceae] & Hamham & C & $\begin{array}{l}\text { Evil eye, Dandruff, } \\
\text { influenza }\end{array}$ & $F, L$ & ZG-056 \\
\hline Datura stramonium L. [Solanaceae] & Mezerbae & $\mathrm{H}$ & $\begin{array}{l}\text { Mange, teeth infection, } \\
\text { stomach pain }\end{array}$ & L, F & ZG-009 \\
\hline $\begin{array}{l}\text { Dichrostachys cinerea (L.) Wight \& Arn. } \\
\text { [Fabaceae] }\end{array}$ & Gonok & Sh & $\begin{array}{l}\text { Sprain, inflammatory } \\
\text { wounds }\end{array}$ & $S, L$ & ZG-005 \\
\hline $\begin{array}{l}\text { Diospyros mespiliformis Hochst. ex A. DC. } \\
\text { [Ebenaceae] }\end{array}$ & Aye & $T$ & Ring worm & $\mathrm{F}$ & ZG-023 \\
\hline $\begin{array}{l}\text { Eucalyptus camaldulensis Dehnh. } \\
\text { [Myrtaceae ] }\end{array}$ & $\begin{array}{l}\text { Keyh- } \\
\text { kelamitos }\end{array}$ & $T$ & $\begin{array}{l}\text { Evil spirit/swells } \\
\text { and wounds }\end{array}$ & L & ZG-031 \\
\hline Eucalyptus globulosus St.-Lag. [Myrtaceae] & $\begin{array}{l}\text { Tsaeda- } \\
\text { kelamitos }\end{array}$ & $\mathrm{T}$ & Cough & L & ZG-025 \\
\hline $\begin{array}{l}\text { Euphorbia candelabrum Tremaut ex Kotschy } \\
\text { [Euphorbiaceae] }\end{array}$ & Kolonqual & $T$ & $\begin{array}{l}\text { Swelling, } \\
\text { spleenomegally }\end{array}$ & ME & ZG-046 \\
\hline Euphorbia tirucalli L. [Euphorbiaceae] & Kinchib & Sh & \begin{tabular}{|l} 
Warts, swelling, \\
internal parasites, \\
petriasis versicolor
\end{tabular} & $\mathrm{ME}$ & ZG-059 \\
\hline $\begin{array}{l}\text { Flueggea virosa (Roxb. ex Willd.) Royle } \\
\text { [Euphorbiaceae] }\end{array}$ & Harmazo & Sh & Sprain, rabies & $\mathrm{R}, \mathrm{Yt}$ & ZG-017 \\
\hline
\end{tabular}




\begin{tabular}{|c|c|c|c|c|c|}
\hline Medicinal plants [Family] & Local name & Habit & Diseases treated & $\begin{array}{l}\text { Parts } \\
\text { used }\end{array}$ & $\begin{array}{l}\text { Voucher } \\
\text { number }\end{array}$ \\
\hline $\begin{array}{l}\text { Gardenia ternifolia Schumach. \& Thonn. } \\
\text { [Rubiaceae] }\end{array}$ & Hatsinay & $T$ & Sprain & S & ZG-021 \\
\hline $\begin{array}{l}\text { Grewia ferruginea Hochst. ex A. Rich. } \\
\text { [Malvaceae] }\end{array}$ & Tsinquayt & Sh & $\begin{array}{l}\text { Leech, Retained } \\
\text { fatal placenta } \\
\text { retention, fire burn }\end{array}$ & $\mathrm{B}, \mathrm{L}$ & ZG-063 \\
\hline $\begin{array}{l}\text { Jasminum abyssinicum Hochst. ex DC. } \\
\text { [Oleaceae] }\end{array}$ & Habi-tselim & $\mathrm{T}$ & Tape worm, vomiting & L & ZG-051 \\
\hline Lepidium sativum L. [Brassicaceae] & Shinfae & $\mathrm{H}$ & $\begin{array}{l}\text { Anthrax, stomach pain, } \\
\text { tonsillitis, diarrhea }\end{array}$ & $\mathrm{F}$ & ZG-035 \\
\hline $\begin{array}{l}\text { Maytenus senegalensis (Lam.) Exell } \\
\text { [Celastraceae] }\end{array}$ & Argudi & $T$ & Stomach pain & B & ZG-026 \\
\hline $\begin{array}{l}\text { Maytenus arbutifolia R. Wilczek } \\
\text { [Celastraceae] }\end{array}$ & At-at & Sh & Sprain & $S$ & ZG-004 \\
\hline Nigella sativa L. [Ranunculaceae] & Awesda & $\mathrm{H}$ & Stomach pain & $\mathrm{F}$ & ZG-033 \\
\hline Olea europaea L. [Oleaceae] & Awlie & $T$ & Colic & $R / L$ & ZG-024 \\
\hline $\begin{array}{l}\text { Ormocarpum pubescens (Hochst.) Cufod. } \\
\text { ex J.B. Gillett [Fabaceae] }\end{array}$ & Alendia & Sh & Wounds & $\mathrm{R}$ & ZG-055 \\
\hline Otostegia integrifolia Benth. [Lamiaceae] & Chiendog & Sh & Insecticide & Wp & ZG-039 \\
\hline Phoenix reclinata Jacq. [Arecaceae] & Siye & $T$ & Evil eye & $\mathrm{R}$ & ZG-016 \\
\hline $\begin{array}{l}\text { Phytolacca dodecandra L'Hér. } \\
\text { [Phytolaccaceae] }\end{array}$ & Shibti & $\mathrm{C}$ & $\begin{array}{l}\text { Rabies, leeches, warts, } \\
\text { herpes virus }\end{array}$ & $\mathrm{R} / \mathrm{F}, \mathrm{L}$ & ZG-038 \\
\hline $\begin{array}{l}\text { Piliostigma thonningii (Schumach.) } \\
\text { Milne-Redh. [Fabaceae] }\end{array}$ & Amam-gemel & $T$ & Rectal prolapse & B & ZG-045 \\
\hline Pluchea dioscoridis (L.) DC. [Asteraceae] & Shitene & $T$ & Anti-vomit & $\mathrm{L}$ & ZG-057 \\
\hline Plumbago zeylanica L. [Plumbaginaceae] & Aftuh & Sh & $\begin{array}{l}\text { Anthrax, teeth infection, } \\
\text { stomach pain/diarrhea }\end{array}$ & $\mathrm{R}$ & ZG-058 \\
\hline Rhamnus prinoides L'Hér. [Rhamnaceae] & Gesho & Sh & $\begin{array}{l}\text { Tonsil, anthrax, } \\
\text { hyper-bilirubinemia }\end{array}$ & $\mathrm{L} / \mathrm{Yt}$ & ZG-002 \\
\hline Ricinus communis L. [Euphorbiaceae] & Gulei & $\mathrm{T}$ & Wounds & $\mathrm{L}$ & ZG-047 \\
\hline Rumex abyssinicus Jacq. [Polygonaceae] & Moqmoqo & $\mathrm{H}$ & $\begin{array}{l}\text { Tuberculosis, teeth } \\
\text { infections, abdominal } \\
\text { problems, stomach pain }\end{array}$ & $\mathrm{R}$ & ZG-034 \\
\hline Ruta chalepensis L. [Rutaceae] & Chena-adam & $\mathrm{H}$ & Evil sprit & $\mathrm{L}$ & ZG-043 \\
\hline Schinus molle L. [Anacardiaceae] & Tikur-berbere & $\mathrm{T}$ & Herpes virus & $\mathrm{L}$ & ZG-052 \\
\hline $\begin{array}{l}\text { Securidaca longipedunculata Fresen. } \\
\text { [Polygalaceae] }\end{array}$ & Shitara & $T$ & $\begin{array}{l}\text { Evil eye, anthrax, } \\
\text { stomach pain, } \\
\text { abdominal problems }\end{array}$ & $\mathrm{R} / \mathrm{B}$ & ZG-061 \\
\hline Senna singueana (Delile) Lock [Fabaceae] & $\begin{array}{l}\text { Hambo- } \\
\text { hambo }\end{array}$ & Sh & $\begin{array}{l}\text { Teeth infection, } \\
\text { stomach pain, sprain }\end{array}$ & $\begin{array}{l}\text { L, R, } \\
B, S\end{array}$ & ZG-010 \\
\hline Sida ovata Forssk. [Malvaceae] & Dekidaero & $\mathrm{H}$ & $\begin{array}{l}\text { Inflammatory } \\
\text { wounds, pus }\end{array}$ & $L, R$ & ZG-020 \\
\hline Solanum incanum L. [Solanaceae] & Engule & $\mathrm{H}$ & $\begin{array}{l}\text { Stomach pain, } \\
\text { swelling, eye diseases, } \\
\text { lumpyskin disease }\end{array}$ & $\begin{array}{l}\text { R, B, } \\
F\end{array}$ & ZG-015 \\
\hline
\end{tabular}




\section{Authors - An Ethnobotanical Study of Medicinal Plants in Asgede Tsimbila District, Northwestern Tigray, Northern Ethiopia}

\begin{tabular}{|c|c|c|c|c|c|}
\hline Medicinal plants [Family] & Local name & Habit & Diseases treated & $\begin{array}{l}\text { Parts } \\
\text { used }\end{array}$ & $\begin{array}{l}\text { Voucher } \\
\text { number }\end{array}$ \\
\hline $\begin{array}{l}\text { Stereospermum kunthianum Cham. } \\
\text { [Bignoniaceae] }\end{array}$ & Adgi-zanay & $\mathrm{T}$ & $\begin{array}{l}\text { Broken/wounded/ } \\
\text { bleeding body part }\end{array}$ & $\mathrm{B}$ & ZG-042 \\
\hline Tamarindus indica L. [Fabaceae] & Humer & $\mathrm{T}$ & $\begin{array}{l}\text { Abdominal problems, } \\
\text { hypertension, } \\
\text { splenomegally }\end{array}$ & $\mathrm{F}$ & ZG-062 \\
\hline Terminalia brownii Fresen. [Combretaceae] & Weyba & $\mathrm{T}$ & $\begin{array}{l}\text { Diarrhoea, hyper- } \\
\text { bilirubinemia }\end{array}$ & $B, L$ & ZG-019 \\
\hline $\begin{array}{l}\text { Tragia cinerea (Pax) M.G. Gilbert } \\
\text { \& Radcl.-Sm. [Euphorbiaceae] }\end{array}$ & Shashito & $\mathrm{C}$ & Sprain, scorpion bite & $R, L$ & ZG-049 \\
\hline $\begin{array}{l}\text { Verbascum sinaiticum Benth. } \\
\text { [Scrophulariaceae] }\end{array}$ & Tirnaka & $\mathrm{H}$ & $\begin{array}{l}\text { Epistaxis/nasal } \\
\text { bleeding, fire burn }\end{array}$ & $\mathrm{F}, \mathrm{L}$ & ZG-053 \\
\hline Withania somnifera (L.) Dunal [Solanaceae] & Agol & Sh & $\begin{array}{l}\text { Swelling/evil spirit, } \\
\text { eye disease }\end{array}$ & $R, L$ & ZG-006 \\
\hline Ximenia americana L. [Ximeniaceae] & Mileo & $\mathrm{T}$ & $\begin{array}{l}\text { Anti-vomit, leech } \\
\text { infestation, tonsillitis }\end{array}$ & $\mathrm{L}$ & ZG-022 \\
\hline Zingiber officinale Roscoe [Zingiberaceae] & Zingible & $\mathrm{H}$ & $\begin{array}{l}\text { Stomach pain, } \\
\text { corneal opacity }\end{array}$ & $\mathrm{R}$ & ZG-007 \\
\hline Ziziphus spina-christi (L.) Desf. [Rhamnaceae] & Gaba & $\mathrm{T}$ & $\begin{array}{l}\text { Dandruff, stomach pain, } \\
\text { tonsil, scorpion bite }\end{array}$ & L, B & ZG-013 \\
\hline
\end{tabular}

mesticated and cultivated near the home, while $7 \%$ are semi-wild. The medicinal plants from the wild are distributed in forest patches, woodlands, shrub lands, rocky hillsides, around grazing and browsing lands, close to stream/river, roadsides, near farmlands, and spiritually protected areas. Cultivated medicinal plants in the area are A. sativum, Aloe vera (L.) Burm.f., Azadirachta indica A. Juss., R. prinoids, R. communis, and Withania somnifera (L.) Dunal.

\section{Informant consensus and preference ranking}

Plumbago zylanica L. was cited by 34 informants (47\%); Solanum incanum L. was the second (28 informants, $39 \%$ ). Anogeissus leiocarpa (DC.) Guill. \& Perr. and W. somnifera ranked third (17 informants, $24 \%$ each). Favorite species in each locality were also reported on the basis of the number of citations given. P. zeylanica (54.2\%) followed by $S$. incanum (41.2\%) are of high preference in Alogien, while $P$. zeylanica $(45.8 \%)$ followed by $W$. somnifera $(29.2 \%)$ are most valued in Lemlem, and in Hintset, S. incanum (54.2\%) followed by $P$. zeylanica $(41.2 \%)$ are preferred.

Six informants selected among the local elders on the basis of the wealth of indigenous knowledge provided their preference ranking of six medicinal plants that are reported to be effective against abdominal disorders (Table 2).

\section{Medicinal plants and their diversity of uses}

Of the total medicinal species, $53(77.9 \%)$ were reported to have multipurpose roles, while $15(22.10 \%)$ have only medicinal values (Table 3). Direct matrix ranking (Table 4) showed that Ziziphus spina-christi (L.) Desf. is the most

Table 2. Preference ranking by six respondents of medicinal plants used for treating abdominal disorders in Asgede Tsimbila District, Tigray Region, Ethiopia.

\begin{tabular}{|c|c|c|c|c|c|c|c|c|}
\hline \multirow[t]{2}{*}{ Plant Species } & \multicolumn{6}{|c|}{ Respondents } & \multirow[t]{2}{*}{ Total } & \multirow[t]{2}{*}{ Rank } \\
\hline & R1 & $\mathbf{R 2}$ & R3 & R4 & R5 & R6 & & \\
\hline Senna singueana (Delile) Lock & 5 & 6 & 5 & 6 & 5 & 6 & 33 & $1 \mathrm{st}$ \\
\hline Solanum incanum $\mathrm{L}$. & 6 & 4 & 6 & 3 & 4 & 5 & 28 & 2nd \\
\hline Zingiber officinale Roscoe & 4 & 5 & 4 & 5 & 6 & 3 & 27 & $3 r d$ \\
\hline Anogeissus leiocarpa Guill. \& Perr. & 2 & 3 & 1 & 4 & 3 & 4 & 17 & 4th \\
\hline Ziziphus spina-christi (L.) Desf. & 3 & 2 & 3 & 1 & 1 & 2 & 12 & 5 th \\
\hline Cucumis dipsaceus Ehrenb. & 1 & 1 & 2 & 2 & 2 & 1 & 9 & 6th \\
\hline
\end{tabular}


Table 3. Service categories of medicinal plants in Asgede Tsimbila District, Tigray Region, Ethiopia.

\begin{tabular}{|l|c|c|}
\hline Service categories & $\begin{array}{c}\text { Number of } \\
\text { species }\end{array}$ & $\begin{array}{c}\text { \% of total } \\
\text { species }\end{array}$ \\
\hline Medicine only & 15 & 22.1 \\
\hline Medicinal plus other uses & 53 & 77.9 \\
\hline Fuel wood and charcoal & 37 & 54.4 \\
\hline Construction & 18 & 26.5 \\
\hline Shade & 16 & 23.5 \\
\hline Food/fruit & 15 & 22.1 \\
\hline Fodder & 14 & 20.6 \\
\hline Fence & 8 & 11.8 \\
\hline Household furniture & 7 & 10.3 \\
\hline Farm implements & 5 & 7.4 \\
\hline Bee forage & 5 & 7.4 \\
\hline Tooth brush & 3 & 4.4 \\
\hline Spice & 2 & 2.9 \\
\hline Gum and resin & 1 & 1.5 \\
\hline
\end{tabular}

preferred medicinal plant by the local people for its various uses, while Cordia africana Lam. was ranked second.

\section{Current status and trends in abundance of medicinal plants}

According to interview results, it is recognized that the vegetation cover of the locality has dramatically changed over time. Results of the vegetation survey (Table 5) showed that Z. spina-christi and Calpurnia aurea (Aiton) Benth. are the most common species each occurring in $14(77.78 \%)$ of the quadrats surveyed. In order to assess trends in abundance of some of the most commonly reported medicinal plants in the district, abundance scores of seven medicinal plants for the period 1970 to 2010 were established (Figure 2).

\section{Knowledge distribution of medicinal plants}

Medicinal plant knowledge based on age and gender is reported in Figure 3. A Pearson correlation test indicated a positive and significant $(r=0.335, p=0.004, \alpha=0.001)$ correlation between age group and the number of species reported by the respondents in the study area.

\section{Threats to conservation status of medicinal plants}

Of five provided threats to medicinal plants (Table 6), agricultural expansion $(26.7 \%)$ is the main threat to wild plant species. Overgrazing was relatively perceived to be least destructive factor $(11.1 \%)$.

\section{Discussion}

\section{Traditional medicinal plants reported and associated knowledge}

The finding of Euphorbiaceae and Fabaceae (six species each), and Cucurbitaceae and Solanaceae (three species each) plant families as the contributor of higher number of species used for medicinal purposes is shared with similar studies elsewhere in Ethiopia (Gidey

Table 4. Average score for direct matrix ranking of seven medicinal plants in Asgede Tsimbila District, Tigray Region, Ethiopia. Use criteria ( $0=$ no use, $1=$ least, $2=$ less, $3=$ good, $4=$ very good and $5=$ excellent).

\begin{tabular}{|c|c|c|c|c|c|c|c|c|c|}
\hline \multirow[t]{2}{*}{ Use categories } & \multicolumn{7}{|c|}{ Plant species scores } & \multirow[t]{2}{*}{ Total } & \multirow[t]{2}{*}{ Rank } \\
\hline & 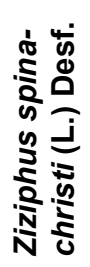 & 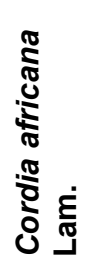 & 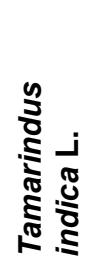 & 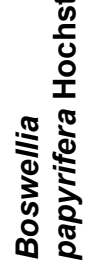 & 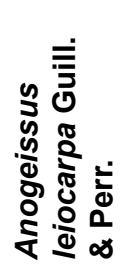 & 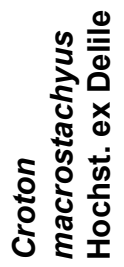 & 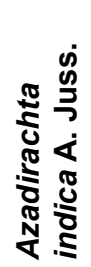 & & \\
\hline Fuel wood & 5 & 4 & 4 & 4 & 4 & 3 & 3 & 27 & $1^{\text {st }}$ \\
\hline Construction & 4 & 5 & 4 & 4 & 3 & 3 & 3 & 26 & \multirow[t]{3}{*}{$2^{\text {nd }}$} \\
\hline Medicine & 4 & 4 & 3 & 3 & 3 & 5 & 4 & 26 & \\
\hline Shade & 4 & 4 & 3 & 3 & 3 & 4 & 5 & 26 & \\
\hline Fodder & 4 & 5 & 2 & 4 & 2 & 0 & 0 & 17 & $5^{\text {th }}$ \\
\hline Fruit/food & 5 & 5 & 4 & 0 & 0 & 0 & 0 & 14 & $6^{\text {th }}$ \\
\hline Fencing & 5 & 0 & 0 & 0 & 0 & 0 & 0 & 5 & $7^{\text {th }}$ \\
\hline Total & 31 & 27 & 20 & 18 & 15 & 15 & 15 & & \\
\hline Rank & $1^{\text {st }}$ & $2^{\text {nd }}$ & $3^{\text {rd }}$ & $4^{\text {th }}$ & \multicolumn{3}{|c|}{$5^{\text {th }}$} & & \\
\hline
\end{tabular}




\section{Authors - An Ethnobotanical Study of Medicinal Plants in Asgede Tsimbila District, Northwestern Tigray, Northern Ethiopia}

Table 5. Most frequently occurring plant species in Asgede Tsimbila District, Tigray Region, Ethiopia. Uses: M (medicinal); E (edible fruits).

\begin{tabular}{|l|l|c|c|c|}
\hline Species & Use & $\begin{array}{c}\text { Positive number of } \\
\text { quadrats with species }\end{array}$ & $\begin{array}{c}\text { Frequency } \\
\text { (\%) }\end{array}$ & $\begin{array}{c}\text { Relative } \\
\text { Frequency }\end{array}$ \\
\hline Ziziphus spina-christi (L.) Desf. & $\mathrm{M}, \mathrm{E}$ & 14 & 77.78 & 5.53 \\
\hline Calpurnia aurea (Aiton) Benth. & $\mathrm{M}$ & 14 & 77.78 & 5.53 \\
\hline Cordia africana Lam. & $\mathrm{M}, \mathrm{E}$ & 13 & 72.2 & 5.14 \\
\hline Croton macrostachyus Hochst. ex Delile & $\mathrm{M}$ & 13 & 72.2 & 5.14 \\
\hline Diospyros mespiliformis Hochst. ex A. DC. & $\mathrm{M}, \mathrm{E}$ & 12 & 66.67 & 4.74 \\
\hline Senna singueana (Delile) Lock & $\mathrm{M}$ & 12 & 66.67 & 4.74 \\
\hline Acacia lahai Steud. \& Hochst. ex Benth. & & 12 & 66.67 & 4.74 \\
\hline Ficus vasta Forssk. & $\mathrm{E}$ & 12 & 66.67 & 4.74 \\
\hline Anogeissus leiocarpa (DC.) Guill. \& Perr. & $\mathrm{M}$ & 11 & 61.11 & 4.35 \\
\hline Dichrostachys cinerea (L.) Wight \& Arn. & $\mathrm{M}$ & 11 & 61.11 & 4.35 \\
\hline Carissa edulis (Forssk). Vahl & $\mathrm{M}, \mathrm{E}$ & 11 & 61.11 & 4.35 \\
\hline Trichilia emetica Vahl & & 11 & 61.11 & 4.35 \\
\hline Ficus sycomorus L. & $\mathrm{E}$ & 11 & 61.11 & 4.35 \\
\hline Flueggea virosa (Roxb. ex Willd.) Royle & $\mathrm{M}, \mathrm{E}$ & 10 & 55.56 & 3.95 \\
\hline Rhus retinorrhoea Steud. ex Oliv. & $\mathrm{E}$ & 10 & 55.56 & 3.95 \\
\hline Albizia amara (Roxb.) Boivin & & 10 & 55.56 & 3.95 \\
\hline Combretum hartmannianum C. Schweinf. & & 9 & 50 & 3.56 \\
\hline Combretum sp. & $\mathrm{M}$ & 9 & 50 & 3.56 \\
\hline Dodonaea angustifolia L.f. & & 8 & 44.4 & 3.16 \\
\hline Tamarindus indica L. & $\mathrm{M}, \mathrm{E}$ & 8 & 44.4 & 3.16 \\
\hline Maytenus arbutifolia R. Wilczek & $\mathrm{M}$ & 7 & 38.89 & 2.77 \\
\hline Balanites aegyptiacus (L.) Delile & $\mathrm{M}, \mathrm{E}$ & 7 & 38.89 & 2.77 \\
\hline Faidherbia albida (Delile) A. Chev. & & 6 & 33.33 & 2.37 \\
\hline Ximenia americana L. & $\mathrm{M}, \mathrm{E}$ & 6 & 33.33 & 2.37 \\
\hline Acacia polyacantha Willd. & & 6 & 33.33 & 2.37 \\
\hline
\end{tabular}

2001, Hunde et al. 2006, Mesfin et al. 2009, Yineger et al. 2008). The number of traditional medicinal plants reported (68 species) and their uses by the local community (Tigryan people) is diverse and includes associated local knowledge on these resources and their applications. This is comparable to other ethnic groups in Ethiopia, i.e., the Gedeo people in southern Ethiopia who use 65 species (Mesfin et al. 2009), the Amhara people in northwestern Ethiopia who use 67 species and the Oromo ethnic group in southwestern Ethiopia who use about 67 species (Yine-

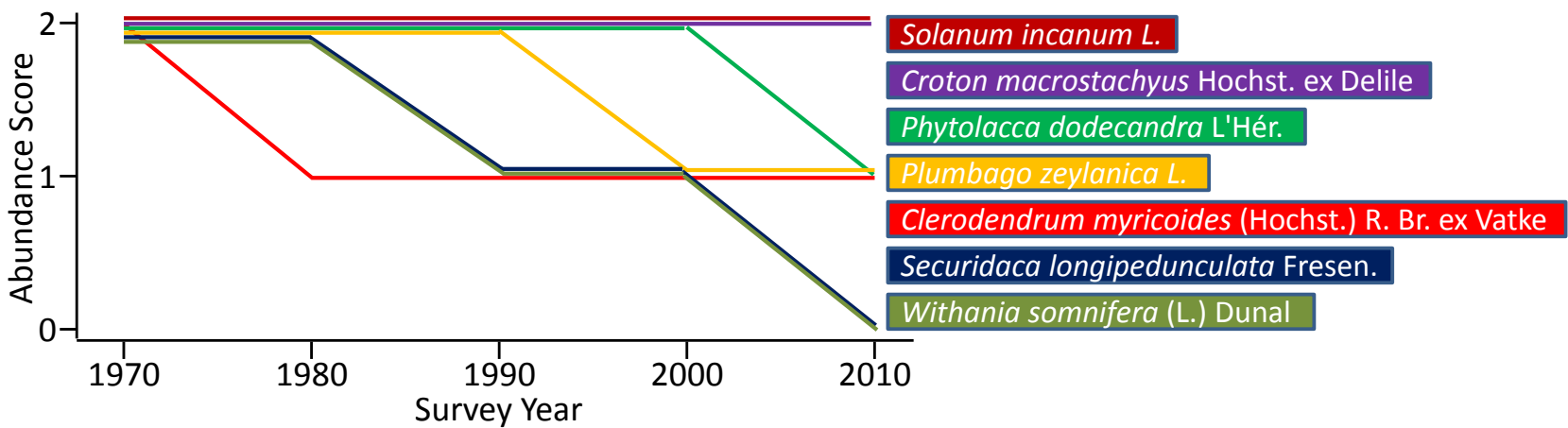

Figure 2. Trends in abundance of most commonly cited medicinal plants in Asgede Tsimbila District, Tigray Region, Ethiopia. Plant species abundance scores: 0 (none or almost none); 1 (a few or some); and 2 (many). 


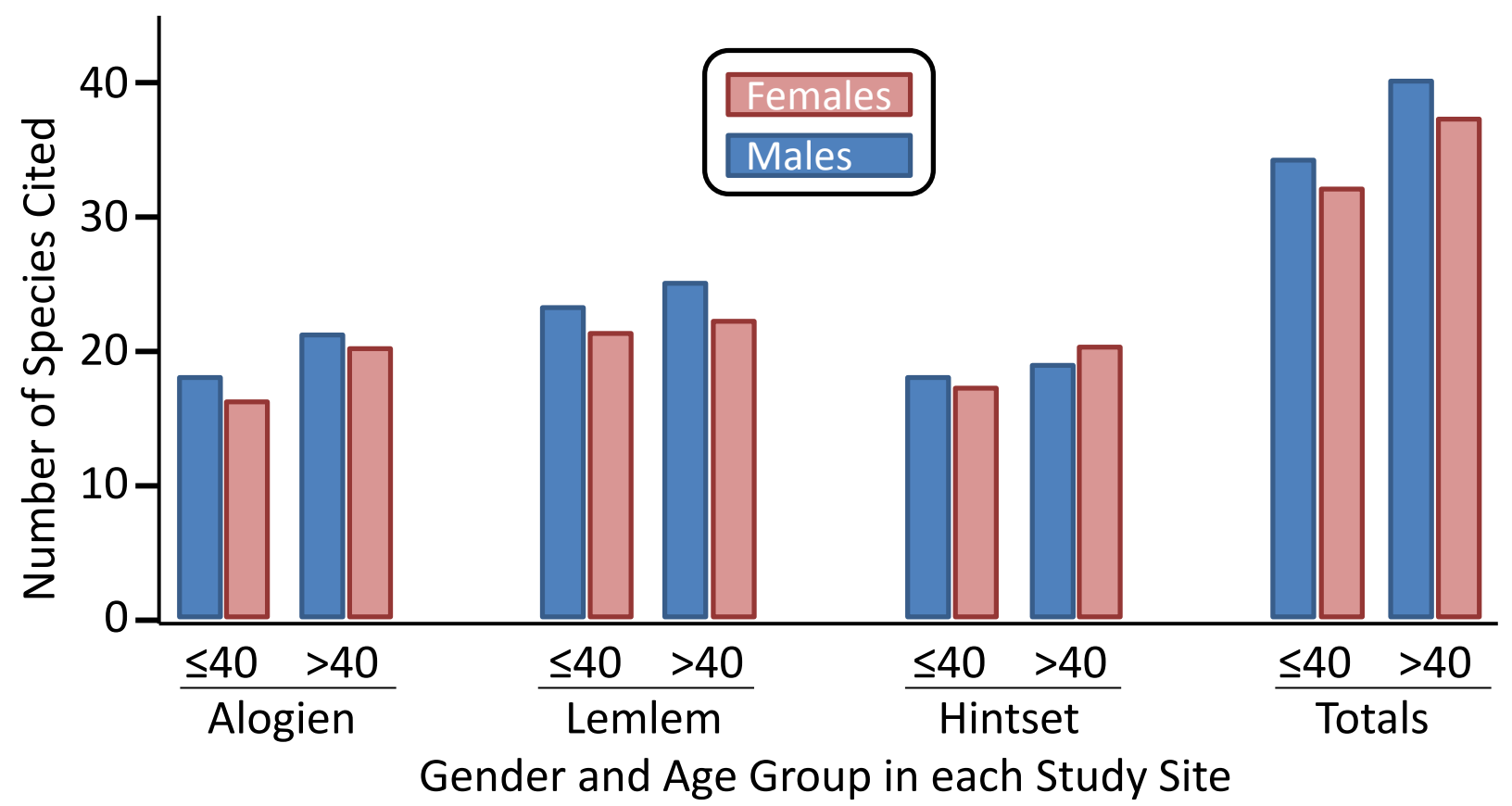

Figure 3. Medicinal plant knowledge base by age and gender in Asgede Tsimbila District, Tigray Region, Ethiopia.

ger et al. 2008). Other studies came up with even higher number of species used among some of the major ethnic groups in the country. For instance, the Oromo people (the major ethnic group in Ethiopia) residing in Mana Angetu district, southeastern Ethiopia make use of 230 species (Lulekal et al. 2008) and the Konta people in southern Ethiopia make use of about 120 species (Hailemariam et al. 2009). On the other hand, the Zay people use relatively fewer species (33) (Gidey 2001). This could be an indication for the assumption that the loss of tradition (cultural shift) in minorities is greater than in larger groups (Luseba \& Van Der Merwe 2006).

Although the dose and mode of application may vary, an appreciable number of the medicinal plants recorded in this study are also medicinally important in other parts of Ethiopia (Amenu 2007, Gidey 2001, Hunde et al. 2006, Teklehaymanot \& Gidey 2007). Some of the plants that were reported in common include; Allium sativum L., Calotropis procera (Aiton) W.T. Aiton, Capparis tomentosa Lam., Carica papaya L., Carissa edulis (Forssk). Vahl,
Clerodendrum myricoides (Hochst.) R. Br. ex Vatke, C. africana, Croton macrostachyus Hochst. ex Delile, Euphorbia tirucali L., Phytolacca dodecandra L'Hér., P. zylanica, Rhamnus prinoides L'Hér., Ricinus communis L., S. incanum, $W$. somnifera and Z. spina-christi. These similarities indicate the wide use of medicinal plant species and the existence of associated knowledge shared among ethnic groups in Ethiopia. The fact that some of the reported plants are having similar uses elsewhere in the world supports their likely pharmacological effectiveness having been tested in different areas by different cultures (Hailemariam et al. 2009).

The medicinal plants were reported as being used for the treatment of about 50 different health problems (28 ailments in humans, 10 in livestock and 12 in both). While the majority of medicinal plants reported were used to treat human health problems (48 species, $70.6 \%$ ), three species $(4.4 \%)$ were used to treat livestock ailments and 17 species $(25 \%)$ were used to treat ailments common to both. The study also showed that the highest propor-

Table 6. Priority ranking ( $1=$ least, $5=$ most) by six respondents of threatening factors based on their level of destructive effects in Asgede Tsimbila District, Tigray Region, Ethiopia.

\begin{tabular}{|c|c|c|c|c|c|c|c|c|c|}
\hline \multirow{2}{*}{$\begin{array}{l}\text { Threatening } \\
\text { Factors }\end{array}$} & \multicolumn{6}{|c|}{ Respondents } & \multirow[t]{2}{*}{ Total } & \multirow[t]{2}{*}{$\%$} & \multirow[t]{2}{*}{ Rank } \\
\hline & R1 & R2 & R3 & R4 & R5 & R6 & & & \\
\hline Agriculture & 4 & 2 & 4 & 5 & 5 & 4 & 24 & 26.7 & $1 \mathrm{st}$ \\
\hline Firewood & 3 & 5 & 5 & 3 & 4 & 3 & 23 & 25.6 & 2nd \\
\hline Drought & 5 & 1 & 3 & 4 & 2 & 5 & 20 & 22.2 & $3 r d$ \\
\hline Construction & 2 & 4 & 2 & 2 & 1 & 2 & 13 & 14.4 & 4th \\
\hline Over-grazing & 1 & 3 & 1 & 1 & 3 & 1 & 10 & 11.1 & 5 th \\
\hline
\end{tabular}




\section{Authors - An Ethnobotanical Study of Medicinal Plants in Asgede Tsimbila District, Northwestern Tigray, Northern Ethiopia}

tion of medicinal plants was used for treating abdominal disorders $(35.3 \%)$, then wounds $(17.6 \%)$ in humans, and anthrax $(5.9 \%)$, followed by mange, leech and epilepsy (4.4\% each) in livestock. The term abdominal disorders, in this context, is a general term for a wide range of bowel (intestinal) related ailments which may be caused by bacteria, protozoa or parasitic worms. Although fewer medicinal plants are used to treat livestock diseases than for humans, most informants reported that in most cases they treat livestock problems with traditional medicine and rarely look for modern medications. This may be attributed to the limited veterinary services in the rural areas, economic factors and the trust the local community developed in these plants treating livestock health problems.

Traditional medicine was preferred to modern medical systems in treating herpes virus (almaz), epilepsy (tezwur), snake bite (niksit temen), evil eye (buda), rabies (himam ebud kelbi), anthrax (megerem), and abdominal disorders (kurtset kebdi). Modern medicine is not recommended at all for herpes virus, with the notion that modern medication for such diseases may have ill effects on the patient. However, no such taboos were reported by the informants associated with the use of traditional medicinal plants, except that there could be ill effects due to the lack of proper dosage.

\section{Habitat, growth forms and plant parts used}

\section{Habitat}

Medicinal plant source habitats in the study area are consistent with the findings of Yineger and Yewhalaw (2007) where most of the medicinal plants $(85.7 \%)$ utilized in southwestern Ethiopia were harvested from the wild. Of the cultivated plants, only $W$. somnifera and $A$. vera were cultivated primarily for their medicinal values. Therefore, wild habitats are the major pool of medicinal plant resources for the local community.

Our observation is in agreement with previous reports from Ethiopia. Medicinal plants cultivated in home gardens were found to be 6\% (Asfaw 1997) and $8.7 \%$ (Tolassa 2007). In the same vein, ethnobotanical study of medicinal plants in Bale Mountains National Park, southeastern Ethiopia (Yineger et al. 2008) and in the central Rift Valley of Ethiopia (Gidey 2001) confirmed that there is little practice of domesticating medicinal plants. The fact that most of the medicinal plants are found in the wild also poses a threat to their existence if habitats are destroyed. This concern is shared by other authors (Hailemariam et al. 2009, Hunde et al. 2006) who have called for a timely intervention for their conservation. There is need for coordinated conservation action, based on both in-situ and ex-situ strategies (Hamilton 2003) through the recognition and accommodation of local community knowledge, interests and priorities (Quansah 2004).

\section{Growth forms}

The results of growth form analysis (Figure 4) implies that majority of the medicinal plants in the study area are woody. This is not surprising since these are the dominant forms in the dry flora of the area. Herbaceous plant forms can not withstand drought and, in most cases, they are likely being over-utilized (including the whole plants). Thus, they were only rarely encountered in the study area.

Contrary to our findings, a relatively higher number of shrubs and herbs were previously reported elsewhere in Ethiopia (Amenu 2007 in western Ethiopia, Hailemariam et al. 2009 in the low lands of southwestern Ethiopia). Similarly, other studies undertaken in Boosat subdistrict, central eastern Ethiopia (Hunde et al. 2006), in Gimbi district, western Ethiopia (Tolassa 2007), in Wonago district, southern Ethiopia (Mesfin et al. 2009), and in Mana Angetu district, southeastern Ethiopia (Lulekal et al. 2008) showed that shrubs, followed by herbs and trees are the most frequently used growth forms. These variations could be attributed to agro-ecological diversity of the country that favors different plant forms, and socio-cultural factors which determine specific knowledge in different communities (Bekele 2007).

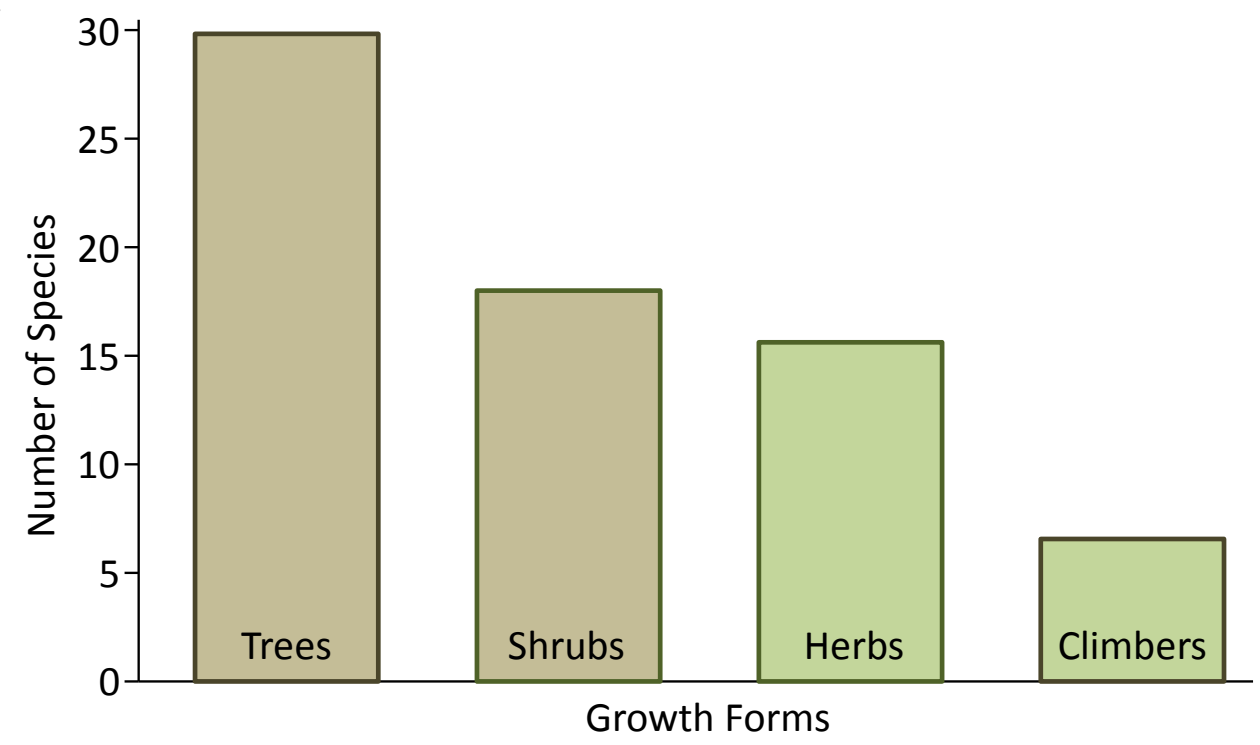

Figure 4. Distribution of medicinal plants by growth forms in Asgede Tsimbila District, Tigray Region, Ethiopia. 


\section{Plant parts used}

With regard to plant parts used medicinally, leaves $(48.5 \%)$ followed by roots $(32.4 \%)$ are the most commonly used. Previous works carried out elsewhere in Ethiopia also revealed that leaves followed by roots were the most common parts (Amenu 2007, Gidey 2001, Hailemariam et al. 2009, Ragunathan \& Solomon 2009). Other reports showed roots to be the most widely used plant parts (Hunde et al. 2006, Lulekal et al. 2008, Mesfin et al. 2009).

Studies have shown that removal of up to $50 \%$ of tree leaves does not significantly affect plant growth (Poffenberger et al. 1992). However, medicinal plant harvest involving roots, rhizomes, bulbs, bark, stems or whole parts have grave consequences both from an ecological point of view and for the survival of the mother plants (Abebe \& Ayehu 1993, Gidey 2001, Hunde et al. 2006). Given that leaves constitute the most frequently sought plant parts in this study, the threat to the destruction of medicinal plants due to plant part extraction appears minimal. Nonetheless, the fact that roots and bark provide the next largest proportion may warrant a possible conservation threat. For example, medicinal plants such as Securidaca longipedunculata Fresen., Aloe sp., C. myricoides and $P$. zeylanica L., which are harvested for their roots, are scarce, perhaps because of medicinal use. Therefore there is need to pay special attention to determine their status and what measures should be taken to ensure their conservation (Gidey 2001).

\section{Informant consensus and preference ranking}

Informant consensus values give good indications about species that serve for particular health problems. Such information underlines the ethnopharmacological significance of medicinal plants in an area (Hailemariam et al. 2009). Some medicinal plants are more popular because they are more familiar to informants being widely recognized due to the wide range of indications in the locality. Other species may have received higher consensus scores due to local abundance and easy access. In general, medicinal plants with higher informant consensus need to be seriously considered for further ethnopharmacological studies since they are species widely applied by many people and probably have been utilized for a long time (Macia et al. 2005). The results imply that species preference within the study sites (sub-districts) did not vary greatly as species distribution, traditional ecological knowledge and economic pursuits of the communities are essentially similar.

Preference ranking (Table 2) showed that Senna singueana (Delile) Lock and S. incanum were ranked first and second thus are considered locally to be the most effective medicinal plants treating abdominal disorders. This is likely due to active anti-microbial substances present within these plants. Therapeutic activity of $S$. incanum ex- tracts has been attributed to the presence of flavonoids, chlorogenics, adenosine, glycopyranoside, solasodine and phenylalkanoic acids (Beaman-Mbaya \& Muhammed 1976, Mwonjoria et al. 2011).

\section{Medicinal plants and their diversity of uses}

Local people in the study area harvest plants with medicinal values for a variety of other uses; mostly for firewood and charcoal, construction, fodder and production of equipment (farm implements and furniture). In line with our results (Table 3 ), Tolassa (2007) reported that $78.82 \%$ of the total medicinal plants used by local people in western Ethiopia have multipurpose roles. The utilization of medicinal plants for variety of added values may result in additional pressures to these resources. This calls for practical solutions like domestication, in-situ conservation, and introduction of other tree species for non-medicinal uses to reduce pressures on the medicinal plants (Tolassa 2007).

In order to assess their relative importance of multi-use plants to the local people and the extent of the existing threats related to their use values, seven common multipurpose species and seven use categories were taken and direct matrix ranking was conducted (Table 4). The seven multipurpose species were selected because they were among the most commonly encountered species, and were reported in equal frequency before the ranking exercise. The results showed that the local people harvest the seven multipurpose species mainly for fuel wood which ranked first. The lowest category is for fencing. These observations may indicate that the sustainability of the top-ranking (most preferred) species may be threatened particularly in view of the daily demand of the local community for these resources (Amenu 2007).

\section{Current status and trends in abundance of medicinal plants}

According to interview results (Table 5), it is likely that the vegetation cover of the locality has changed over time. Obviously, there is a chance of finding plant species either clumped together in one spot or evenly distributed in a given area. Similarly, this study revealed that about $60 \%$ of the most frequent plants in the district are medicinal plants (Table 5). Nevertheless, medicinal plants that received the highest informant consensus are not frequently distributed in the district. This is consistent with Tolassa (2007) where some medicinal plants (e.g., Warburgia ugandensis Sprague) were reported to be less frequently distributed in the face of over utilization and habitat destruction even though they received the highest informant consensus among indigenous people in Gimbi district, western Ethiopia. A similar study undertaken in the central rift valley of Ethiopia (Gidey, 2001) has also reported the abundance of valuable medicinal plants (e.g., S. longepedunculata) to be decreasing with time. These observa- 


\section{Authors - An Ethnobotanical Study of Medicinal Plants in Asgede Tsimbila District, Northwestern Tigray, Northern Ethiopia}

tions indicate that valuable medicinal plants in different parts of the country are being depleted. This could be due to the fact that they are over utilized for diversity of uses and disappeared from some parts, or that the destruction of their habitat through deforestation restricted their occurrence to only some sites.

Abundance scores for seven medicinal plants (Figure 3) show five species have declined with time. Only two remained constant within the given period. Therefore, the abundances of a majority of the medicinal plants declined within the given period. Some of these (e.g., W. somnifera and S. longepedunculata) have shown dramatic declines. The decreasing trend in abundance of medicinal plants could be attributed to the fact that most of the species are heavily harvested because of the multiple uses they have. A similar observation was made in Uganda by Nanyunja (2003) where he stated that the trend in abundance of medicinal plants could be related with the multiple uses of the species.

In general, wild vegetation resources including medicinal plants in different parts of the country are continuously shrinking. The loss of medicinal plants could be due to multiple factors such as deforestation (expansion of agriculture), charcoal production, cutting trees for construction, and fire wood collection, among others. These resources are openly accessed. Repeated drought incidences and poverty (the lack of livelihood options) have aggravated the situation. Drought in the 1970s was mentioned by informants as a major historical event which contributed to the degradation of plant resources.

\section{Knowledge distribution of medicinal plants}

Knowledge distribution of medicinal plants was compared between those $\leq 40$ years and $>40$ years, and between gender (Figure 4). Those older than 40 years tended to cite a higher number of medicinal plants. This is comparable with the findings of Gidey (2001) who found that those above 40 identified more medicinal plants among the Zay people of Ethiopia. A similar observation was also reported by Hunde et al. (2006) and Tolassa (2007). A higher number of species was also reported by males in each locality.

Gidey et al. (2009) also a significantly higher number of medicinal plants are reported by informants above 40 years of age among the Bench ethnic group. However, Yineger and Yewhalaw (2007) reported a statistically insignificant correlation between age and the number of medicinal plants by the local people in Jimma zone, south western Ethiopia. This could be related to knowledge acquisition by the lower age class in that locality. In the present study, the reason elders are more knowledgeable is due to their personal experiences using these plants. The difference in the perception of the two age classes concerning the utilization of traditional medicinal plants will likely result in the loss of plant lore over time (Abebe et al. 2003, Hunde et al. 2004).

No significant correlation between gender and the number of species reported was found $(r=-0.034, p=0.778$, $\alpha=0.05)$. A similar observation was reported on Himalayan medicinal plants (India) (Bisht et al. 2006). In contrast, Gidey et al. (2009) reported a positive and significant correlation between gender and the number of species reported among the Bench ethnic group. In general, various studies in different areas in the country demonstrated the existence of knowledge variations and similarities among social groups. This could be attributed to variation in the cultural and socio-economic aspects of each social group.

\section{Threats to conservation status of medicinal plants}

The study revealed that medicinal plants are under pressure from various anthropogenic and natural factors. Local people's perceptions of factors threatening the medicinal plants were determined through priority ranking among some selected informants (Table 6). Pressures from agricultural expansion, wide spread cutting for fuel wood combined with seasonal drought have been reported by Balemie et al. (2004), Mesfin et al. (2009) and Lulekal et al. (2008) as main factors for environmental degradation as well as the depletion of medicinal plants. In general, several studies in different parts of Ethiopia have shown that wild plant resources including medicinal plants are subjected to a number of anthropogenic and natural factors such as agricultural expansion, collection for fuel and construction, recurrent drought and overgrazing.

As for conservation status, most of the medicinal plants in the study area have no protection since they are harvested from the wild with no evident conservation practices. The few cultivated medicinal plants (e.g., P. zeylanica, $W$. somnifera and Aloe sp.) are being conserved near homes. This urges the need for participation of local people and awareness creation through training or education on sustainable utilization and management of plant resources in general and the medicinal plants in particular.

\section{Conclusion and Recommendations}

The study revealed that the area harbors a diversity of medicinal plants, and associated knowledge. Local people depend on medicinal plants to meet their basic health care needs. The dependency on these plant resources is associated with easy access, perceived efficacy, and cultural values attached to the plants. Medicinal plants are also sources of added value (e.g., wood extraction for fuel and construction, fence, fodder and production of different equipment). Majority of the medicinal plants in the district are woody species (trees and shrubs) that are collected mainly for their leaves. $P$. zeylanica, $S$. incanum, $A$. leiocarpa, $W$. somnifera and $S$. longepedunculata are the most commonly reported medicinal plants in the subdis- 
trict. Age is an important factor responsible for ethnomedicinal knowledge variations with older people being more knowledgeable.

Abundance of medicinal plants is declining because of both anthropogenic and natural factors. Knowledge of medicinal plant use is probably declining through failure to transfer from the elderly to the young due to fading interest of the young. The main threats for these resources emanates from agricultural expansion, wood extraction, recurrent drought, and overgrazing. Conservation efforts are poor. Consequently, plant species will likely be lost unless timely conservation measures are launched. In this regard, public awareness and community based management activities need to be encouraged. Attention should also be geared towards research and development on various aspects of the plants such as biology, ecology, silviculture and management of the species and phytochemical activities.

\section{Acknowledgements}

We are greatly indebted to the local people of Asgede Tsimbila district who generously shared us their wisdom and experiences on traditional medicinal plants. We would like to extend our gratitude to the local administration and rural development offices in the district for facilitating field data collection. The authors also thank anonymous reviewers for their valuable comments.

\section{Literature Cited}

Abebe, D. \& A. Ayehu. 1993. Medicinal Plants and Enigmatic Health Practice of North Ethiopia. Berhanina Selam Printing Enterprise, Addis Ababa.

Abebe, D., A. Debella \& K. Urga. 2003. Medicinal plants and Other Useful Plants of Ethiopia. Camerapix Publishers International, Singapore.

Amenu, E. 2007. Use and Management of Medicinal Plants by Indigenous People of Ejaji Area (Chelya district) West Shoa, Ethiopia: An Ethnobotanical approach. M.Sc thesis, Addis Ababa University, Addis Ababa.

Asfaw, Z. 1997. Indigenous African Food Crops and Useful Plants: Survey of indigenous food crops, their preparations and home gardens. The United Nation University Institute for Natural Resources in Africa, Nairobi.

Balemie, K., E. Kelbessa \& Z. Asfaw. 2004. Indigenous medicinal utilization, management and threats in Fentale Area, Eastern Shewa, Ethiopia. Ethiopian Journal of Biological Science 3(1):1-7.
Beaman-Mbaya, V. \& S.I. Muhammed. 1976. Antibiotic action of Solanum incanum (Linnaeus). Antimicrobial Agents and Chemotherapy 9(6):920-924.

Bekele-Tesemma, A., A. Birnie \& B. Tengnäs. 1993. Useful Trees and Shrubs in Ethiopia: Identification and management. Embassy of Sweden, Regional Soil Conservation Unit/SIDA, Nairobi.

Bekele, E. 2007. Study on Actual Situation of Medicinal Plants in Ethiopia. Prepared for Japan Association for International Collaboration of Agriculture andForestry, Addis Ababa.

Bisht, A.K., A. Bhatt, R.S. Rawal \& U. Dhar. 2006. Prioritization and conservation of Himalayan medicinal plants: Angelica glauca Edgew. as a case study. Ethnobotany Research and Applications 4:011-023.

Cotton, C.M. 1996. Ethnobotany: Principles and applications. John Wiley and Sons Ltd. Chichester, England.

Defar, G. 1998. Non-Wood Forest Products. Pg.83 in Data Collection and Analysis for Sustainable Forest Management in ACP countries Linking National and International Efforts. UNEP, Nakuru, Kenya.

Edwards, S., T. Mesfin \& I. Hedberg. 1995. Editors of Flora of Ethiopia and Eritrea. Volume 2, Part 2, Canellaceae to Euphorbiaceae. The National Herbarium, Addis Ababa University, Addis Ababa.

Edwards, S., T. Mesfin, D. Sebsebe \& I. Hedberg. 2000. Editors of Flora of Ethiopia and Eritrea. Volume 2, Part 1. Magnoliaceae to Flacourtiaceae. The National Herbarium Addis Ababa University, Addis Ababa.

Edwards, S., D. Sebsebe \& I. Hedberg. 1997. Alliaceae. Pp. 148-156 in Flora of Ethiopia and Eritrea. Volume 6. The National Herbarium, Addis Ababa University, Addis Ababa.

Fichtl, R. \& A. Admasu. 1994. Honey Bee Flora of Ethiopia. Deutscher Entwicklungsdienst, Margraf Verlag, Weikersheim, Germany.

Genzebu, D. 2009. On-Farm Phenotypic Characterization of Arado Cattle Breed in North West Zone of Tigray, Ethiopia. M.Sc thesis, Addis Ababa University, Addis Ababa.

Gidey, M. 2001. An ethnobotanical study on medicinal plants used by the Zay people in Ethiopia. Centrum för Biologisk Mångfald Skriftserie 3:81-99.

Gidey, M., Z. Asfaw, Z. Woldu \& T. Teklehaymanot. 2009. Medicinal plant knowledge of the Bench ethnic group of Ethiopia: Ethnobotanical investigation. Journal of Ethnobiology and Ethnomedicine 5:34. 


\section{Authors - An Ethnobotanical Study of Medicinal Plants in Asgede Tsimbila District, Northwestern Tigray, Northern Ethiopia}

Hailemariam, T., S. Demissew \& Z. Asfaw. 2009. An ethnobotanical study of medicinal plants used by local people in the lowlands of Konta Special Woreda, southern nations, nationalities and peoples regional state, Ethiopia. Journal of Ethnobiology and Ethnomedicine 5:26.

Hamilton, A. 2003. Medicinal plants and conservation: Issues and approaches. Medicinal Plants Specialist Group, IUCN, International Plants Conservation Unit, WWF-UK.

Hedberg, I. \& S. Edwards. 1989. Editors of Flora of Ethiopia and Eritrea. Volume 3, Pittosoraceae to Araliaceae. The National Herbarium, Addis Ababa University, Addis Ababa.

Hedberg, I. \& S. Edwards. 1995. Editors of Flora of Ethiopia and Eritrea. Volume 7, Poaceae (Graminaceae). The National Herbarium, Addis Ababa University, Addis Ababa.

Hedberg, I., K. Ensermu, S. Edwards, D. Sebsebe \& E. Persson. 2006. Editors of Flora of Ethiopia and Eritrea. Volume 5, Plantaginaceae. The National Herbarium, Addis Ababa University, Addis Ababa.

Hedberg, I., I. Friis \& S. Edwards. 2004. Editors of Flora of Ethiopia and Eritrea. Volume 4, Part 2 Asteraceae. The National Herbarium, Addis Ababa University, Addis Ababa.

Hunde, D., Z. Asfaw \& E. Kelbessa. 2006. Use of traditional medicinal plants by people of 'Boosat' sub district, Central Eastern Ethiopia. Ethiopian Journal of Health Science 16(2):141-155.

Hunde, D., Z. Asfaw \& E. Kelbessa. 2004. Use and management of ethnoveterinary medicinal plants by indigenous people in 'Boosat', Wolench. Ethiopian Journal of Biological Science 3(2):113-132.

Kahsay, H. 2007. Assessment of Spatial Distribution, Regenerating and Degradations of Boswellia papyrifera (summarized document for briefing, 29-30/06/2006). Environmental Protection, Land Administration and Use Authority of Tigray, Tigray, Ethiopia.

Lulekal, E., E. Kelbessa, T. Bekele \& H. Yineger. 2008. An ethnobotanical study of medicinal plants in Manna Angetu District, southeastern Ethiopia. Journal of Ethnobiology and Ethnomedicine 4:10.

Luseba, D. \& D. Van Der Merwe. 2006. Ethnoveterinary medicine practices among Tsonga speaking people of South Africa. Onderstepoort Journal of Veterinary Research 73:115-122.

Macia, M.J., E. Garcia \& P.J. Vidaurre. 2005. An ethnobotanical survey of medicinal plants commercialized in the markets of La Paz and El Alto, Bolivia. Journal of Ethnopharmacology 97(2):337-350.

Martin, G.J. 1995. Ethnobotany: A method manual. Chapman and Hall, London.

Mesfin, F., S. Demissew \& T. Teklehaymanot. 2009. An ethnobotanical study of medicinal plants in Wonago Woreda, SNNPR, Ethiopia. Journal of Ethnobiology and Ethnomedicine 5:28.

Mintsa-Mi Nzue, A.P. 2009. Use and Conservation Status of Medicinal Plants in the Cape Peninsula, Western Cape Province of South Africa. M.Sc thesis, University of Stellenbosch, Stellenbosch, South Africa.

Mwonjoria, J.K., H.N. Kariuki \& F.N. Waweru. 2011. The antinociceptive antipyretic effects of Solanum incanum (Linneaus) in animal models. International Journal of Phytopharmacology 2(1): 22-26.

Nanyunja, R.K. 2003. Indigenous Knowledge of the Abundance of Medicinal and Food Plants in Mount Moroto Forest Reserve. Institute of Environmental \& Natural Resources, Makerere University, Kampala, Uganda.

November, E., R. Aerts, M. Behailu \& B. Muys. 2002. Species List Tigrinya- Scientific technical note, 2002/4. Forest Rehabilitation Project, Mekelle University, Ethiopia.

Poffenberger, M., B. McGean, N.H. Ravindranath \& M. Gadgil.1992. Editors of Field Methods Manual Volume 1: Diagnostic tools for supporting joint forest management systems. Society for Promotion of Wastelands Development, New Delhi, India.

Quansah, N. 2004. The neglected key to successful biodiversity conservation and appropriate development: local traditional knowledge. Ethnobotany Research and Applications 2:89-91.

Ragunathan, M. \& M. Solomon. 2009. Ethnomedicinal survey of folk drugs used in Bahirdar Zuria district, north western Ethiopia. Indian Journal of Traditional Knowledge $8(2): 281-284$.

SPSS. 2008. SPSS 16.0. SPSS Inc., Chicago, Illinois.

Teklehaymanot, T. \& M. Gidey. 2007. Ethnobotanical study of medicinal plants used by people in Zegie Peninsula, Northwestern Ethiopia. Journal of Ethnobiology and Ethnomedicine 3:12.

Tolassa, E. 2007. Use and Conservation of Traditional Medicinal Plants by Indigenous People in Gimbi Woreda, Western Wellega, Ethiopia. M.Sc thesis, Addis Ababa University, Addis Ababa. 
Yineger, H., E. Kelbessa, T. Bekele \& E. Lulkal. 2008. Plants used in traditional management of human ailments at Bale Mountains National Park, Southeastern Ethiopia. Journal of Medicinal Plants Research 2(6):132-153.
Yineger, H. \& D. Yewhalaw. 2007. Traditional medicinal plant knowledge and use by local healers in Sekoru District, Jimma Zone, Southwestern Ethiopia. Journal of Ethnobiology and Ethnomedicine 3:24. 